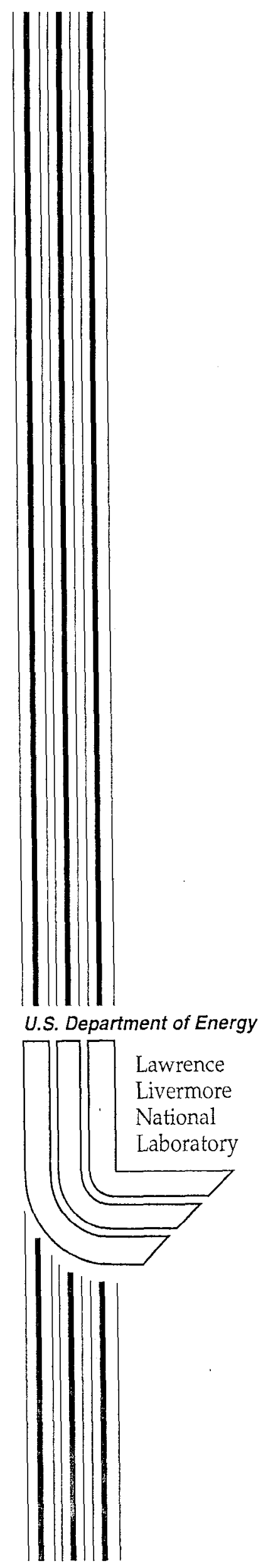

UCRL-ID-138726

\title{
Domain Green's function Sampling in Diffusion Monte Carlo
}

\author{
M.H. Kalos
}

April 10, 2000 


\section{DISCLAIMER}

This document was prepared as an account of work sponsored by an agency of the United States Government. Neither the United States Government nor the University of California nor any of their employees, makes any warranty, express or implied, or assumes any legal liability or responsibility for the accuracy, completeness, or usefulness of any information, apparatus, product, or process disclosed, or represents that its use would not infringe privately owned rights. Reference herein to any specific commercial product, process, or service by trade name, trademark, manufacturer, or otherwise, does not necessarily constitute or imply its endorsement, recommendation, or favoring by the United States Government or the University of California. The views and opinions of authors expressed herein do not necessarily state or reflect those of the United States Government or the University of California, and shall not be used for advertising or product endorsement purposes.

Work performed under the auspices of the U. S. Department of Energy by the University of California Lawrence Livermore National Laboratory under Contract W-7405-Eng-48.

This report has been reproduced

directly from the best available copy.

Available to DOE and DOE contractors from the

Office of Scientific and Technical Information

P.O. Box 62, Oak Ridge, TN 37831

Prices available from (423) 576-8401

http://apollo.osti.gov/bridge/

Available to the public from the

National Technical Information Service

U.S. Department of Commerce

5285 Port Royal Rd.,

Springfield, VA 22161

http://www.ntis.gov/

OR

Lawrence Livermore National Laboratory

Technical Information Department's Digital Library

http://www.llnl.gov/tid/Library.html 


\title{
Domain Green's function Sampling in Diffusion Monte Carlo
}

\author{
M.H. Kalos \\ Lawrence Livermore National Laboratory Livermore CA 94551-0808
}

\begin{abstract}
We discuss the mathematical basis of sampling diffusive paths in Monte Carlo using Green's functions that are themselves built up stochastically from Green's functions in geometrical subdomains. The method of spheres is a special case. We show that other subdomains can be used as well, and may be more efficient for some applications. We include the basis for construction of such subdomain Green's functions for rectangular domains (in any number of dimensions) and cylindrical domains.
\end{abstract}

\section{INTRODUCTION}

Sampling diffusive paths in complicated geometries has a number of diverse applications. It arises in problems of heat conduction which may be treatcd by Montc Carlo, in the diffusion of radiation or chemical species, and in quantum Monte Carlo, especially in the computation of hard-sphere systems [1]. While there are rather straightforward approximate methods available, we will be concerned with exact and efficient methods that rely on the recursive stochastic generation that invoke integrals equations for Green's functions.

We consider the diffusion equation with a spatially dependent absorption rate $A(\vec{R})>0$ and a source of particles that emits $Q(\vec{R}, t)$ per unit time at time $\mathrm{t}$ and position $\vec{R} \in \Omega$ in which the solution $\rho(\vec{R}, t)$ is to be found.

$$
\left[-\nabla^{2}+A(\vec{R})+\frac{\partial}{\partial t}\right] \rho(\vec{R}, t)=Q(\vec{R}, t)
$$

Green's function for this equation is the solution of

$$
\begin{aligned}
{\left[-\nabla^{2}+A(\vec{R})+\frac{\partial}{\partial t}\right] G\left(\vec{R}, \vec{R}_{0} ; t\right) } & =0 ; \quad \vec{R}, \vec{R}_{0} \in \Omega \\
G\left(\vec{R}, \vec{R}_{0} ; 0\right) & =\delta\left(\vec{R}-\vec{R}_{0}\right) . \\
G\left(\vec{R}, \vec{R}_{0} ; t\right) & =0 ; \quad \vec{R}, \vec{R}_{0} \notin \Omega .
\end{aligned}
$$

A formal solution of $\mathrm{Eq}(1.1)$ that uses $G\left(\vec{R}, \vec{R}_{0} ; t\right)$ is

$$
\rho(\vec{R}, t)=\int_{0}^{\infty} \int_{\Omega} G\left(\vec{R}, \vec{R}^{\prime} ; t-t^{\prime}\right) Q\left(\vec{R}^{\prime}, t^{\prime}\right) d \vec{R}^{\prime} d t
$$

If $G\left(\vec{R}, \vec{R}_{0} ; t\right)$ were known analytically, then Eq (1.3) would permit an analytic solution for some forms of $Q(\vec{R}, t)$. Alternatively, if a method for sampling $G\left(\vec{R}, \vec{R}_{0} ; t\right)$ can be found, then 
one may sample a time $t^{\prime}$ and a position $\vec{R}^{\prime}$ from the function $Q\left(\vec{R}^{\prime}, t^{\prime}\right)$ and then, conditional on those coordinates, sample $t$ and $\vec{R}$ from $G\left(\vec{R}, \vec{R}^{\prime} ; t-t^{\prime}\right)$. Unfortunately, except for some special cases, $G$ is not generally known in interesting geometries. Our procedure can be considered as a method for sampling $G$ in a recursive way, so that the second of the two possibilities can be carried through.

\section{RECURSIVE COMPUTATION OF $G$}

Divide the space $\Omega$ into two parts, $\Omega_{0}$, and the complement, $\Omega_{1}$ so that $\Omega=\Omega_{0} \cup \Omega_{1}$. The boundary of $\Omega_{0}$ is denoted by $\partial \Omega_{0}$. A diffusing object (which we will call a "walker") that starts at $\vec{R}_{0} \in \partial \Omega_{0}$ must cross $\partial \Omega_{0}$ if it is to arrive at some position in $\Omega_{1}$. We label the walkers in two ways distinguishing those that have not yet crossed $\partial \Omega_{0}$ from those that have. The property of "not yet crossed" or "NYC" is lost at the boundary, so that walkers labeled NYC are absorbed at the boundary (and then reemitted as the other kind of walker that can diffuse anywhere including back into $\Omega_{0}$. $)$ Let $G_{0}\left(\vec{R}, \vec{R}_{0}, t\right)$ be the distribution of the NYC walkers; it satisfies:

$$
\begin{aligned}
{\left[-\nabla^{2}+A(\vec{R})+\frac{\partial}{\partial t}\right] G_{0}\left(\vec{R}, \vec{R}_{0} ; t\right) } & =0 ; \quad \vec{R}, \vec{R}_{0} \in \Omega_{0} ; \\
G_{0}\left(\vec{R}, \vec{R}_{0} ; 0\right) & =\delta\left(\vec{R}-\vec{R}_{0}\right) ; \\
G_{0}\left(\vec{R}, \vec{R}_{0} ; t\right) & =0 ; \quad \vec{R}, \vec{R}_{0} \notin \Omega .
\end{aligned}
$$

Integrating $\mathrm{Eq}(2.1)$ over $\Omega_{0}$ and using Green's theorem, we find that

$$
\int_{0}^{\infty} \int_{\Omega_{0}} A(\vec{R}) G_{0}\left(\vec{R}, \vec{R}_{0} ; t\right) d \vec{R} d t+\int_{0}^{\infty} \int_{\partial \Omega_{0}}\left[-\vec{n} \cdot \vec{\nabla} G_{0}\left(\vec{R}, \vec{R}_{0} ; t\right)\right] \vec{R} d t=1
$$

Here, $\vec{n}$ is a unit vector outer normal to $\partial \Omega_{0}$.

The first term of the last equation is clearly the net absorption of NYC walkers and the second term gives their net leakage across the boundary, $\partial \Omega_{0}$. Thus we may interpret the expression $-\vec{n} \cdot \vec{\nabla} G_{0}\left(\vec{R}, \vec{R}_{0} ; t\right)$, the outer normal derivative of $G_{0}$ as the current of walkers that arrive at the boundary at position $\vec{R}$ and time $t$ given that they were born at $\vec{R}_{0}$ at time zero.

Since the NYC walkers do in fact continue to diffuse after reaching the boundary of $\Omega_{0}$, we may correctly regard the leakage current as a source for future diffusion. On that basis, and using $\mathrm{Eq}$ (1.3) we immediately conclude that

$$
G\left(\vec{R}, \vec{R}_{0} ; t\right)=G_{0}\left(\vec{R}, \vec{R}_{0} ; t\right)+\int_{0}^{t} \int_{\partial \Omega_{0}} G\left(\vec{R}, \vec{R}^{\prime} ; t-t^{\prime}\right)\left[-\vec{n} \cdot \vec{\nabla} G_{0}\left(\vec{R}^{\prime}, \vec{R}_{0} ; t^{\prime}\right)\right] d \vec{R} d t^{\prime}
$$

This equation may also be proved in a more formal way by multiplying Eq (1.2) by $G_{0}\left(\vec{R}, \vec{R}_{0} ; t\right)$, Eq $(2.1)$ by $G\left(\vec{R}, \vec{R}_{0} ; t\right)$ subtracting, integrating, and again using Green's theorem.

In fact, solving $\mathrm{Eq}(2.1)$ is no easier than solving (1.2) so that the relation in $\mathrm{Eq}(2.3)$, while correct, is of no practical use. But we may proceed as follows. For every point $\vec{R}_{k}$ in $\Omega$ associate a domain $\Omega_{k}$. Let $U_{k}$ be an upper bound to the function $A(\vec{R})$ on $\Omega_{k}$. That is 


$$
U_{k} \geq A(\vec{R}) \quad \vee \vec{R} \in \Omega_{k}
$$

Now define a new Green's function in which $U_{k}$ is the absorption rate:

$$
\begin{aligned}
{\left[-\nabla^{2}+U_{k}+\frac{\partial}{\partial t}\right] G_{k}\left(\vec{R}, \vec{R}_{k} ; t\right) } & =0 ; \quad \vec{R}, \vec{R}_{k} \in \Omega_{k} ; \\
G_{k}\left(\vec{R}, \vec{R}_{k} ; 0\right) & =\delta\left(\vec{R}-\vec{R}_{k}\right) ; \\
G_{k}\left(\vec{R}, \vec{R}_{k} ; t\right) & =0 ; \quad \vec{R}, \vec{R}_{k} \notin \Omega .
\end{aligned}
$$

As we will see presently, there are useful classes of domains for which $G_{k}\left(\vec{R}, \vec{R}_{k} ; t\right)$ satisfying Eq (2.5) can be found analytically, and to sample in the Montc Carlo sense. They overestimate the rate of absorption, but that is easy to correct: Walkers are absorbed at $\vec{R}$ in $\Omega_{k}$ at a rate $U_{k} G_{k}\left(\vec{R}, \vec{R}_{k} ; t\right)$. If with probability $\left[U_{k}-A(\vec{R})\right] / U_{k} \geq 0$ the walkers are reemitted after an artificial absorption at $\vec{R}$, then the true absorption rate is exactly right. These reemitted walkers are also source particles for future diffusion so that the full recursion for $G\left(\vec{R}, \vec{R}_{k} ; t\right)$ is now

$$
\begin{aligned}
G\left(\vec{R}, \vec{R}_{k} ; t\right)=G_{k}\left(\vec{R}, \vec{R}_{k} ; t\right)+\int_{0}^{t} \int_{\partial \Omega_{k}} G\left(\vec{R}, \vec{R}^{\prime} ; t-t^{\prime}\right)\left[-\vec{n} \cdot \vec{\nabla} G_{k}\left(\vec{R}^{\prime}, \vec{R}_{k} ; t^{\prime}\right) d \vec{R}^{\prime} d t^{\prime}\right. \\
+\int_{0}^{t} \int_{\Omega_{k}} G\left(\vec{R}, \vec{R}^{\prime} ; t-t^{\prime}\right) \frac{U_{k}-A\left(\vec{R}^{\prime}\right)}{U_{k}} U_{k} G_{k}\left(\vec{R}^{\prime}, \vec{R}_{k} ; t^{\prime}\right) d \vec{R}^{\prime} d t^{\prime}
\end{aligned}
$$

This equation may be derived more formally as indicated above for $\mathrm{Eq}$ (2.3), and may be verified by operating term by term on both sides with

$$
-\nabla^{2}+A(\vec{R})+\frac{\partial}{\partial t} \equiv-\nabla^{2}+U_{k}+\left[A(\vec{R})-U_{k}\right]+\frac{\partial}{\partial t}
$$

\section{RANDOM WALKS AND INTEGRAL EQUATIONS}

We now digress to establish the relationship of random walks and the kind of recursions for the Green's functions that we have developed.

Let $T(X \mid Y)$ be a stochastic kernel. That is, it satisfies

$$
\begin{array}{r}
T(X \mid Y) \geq 0 \\
\int T(X \mid Y) d X \leq 1 \quad \forall Y
\end{array}
$$

and the smallest eigenvalue $\lambda_{0}$ of $T$ defined by

$$
\varphi_{m}(X)=\lambda_{m} \int T(X \mid Y) \varphi_{m}(Y) d Y
$$

satisfies

$$
\lambda_{0}>0
$$


Now let a random walk be defined by the sequence of steps $\left(Y, X_{1}, X_{2}, \cdots\right)$ chosen as follows:

$$
X_{1} \text { is sampled from } T\left(X_{1} \mid Y\right) \text { conditional on } Y
$$

$X_{2}$ is sampled from $T\left(X_{2} \mid X_{1}\right)$ conditional on $X_{1} \quad \ldots$

$$
X_{m} \text { is sampled from } T\left(X_{m} \mid X_{m-1}\right) \text { conditional on } X_{m-1}
$$

Define $K_{m}(Z \mid Y)$ to be the density of arrivals of walkers near $Z$ after exactly $m$ steps starting from $Y$ following 3.4. Then

$$
\begin{aligned}
K_{1}(Z, Y) & =T(Z \mid Y) \\
K_{2}(Z, Y) & =\int T(Z \mid X) T(X \mid Y) d X \quad \cdots \\
K_{m}(Z \mid Y) & =\int \cdots \int T\left(Z \mid X_{m-1}\right) T\left(X_{m-1} \mid X_{m-2}\right) \cdots T\left(X_{1} \mid Y\right) d X_{1} \cdots d X_{m-1} \\
& =\int K_{m-1}(Z \mid X) T(X \mid Y) d X
\end{aligned}
$$

Let $K(Z \mid Y)$ be the the density of arrivals of walkers near $Z$ after any number of steps. It is simply

$$
K(Z \mid Y)=\sum_{1}^{\infty} K_{m}(Z \mid Y)
$$

But

$$
\int K(Z \mid X) T(X \mid Z) d X=\sum_{2}^{\infty} K_{m}(Z \mid Y)=K(Z \mid Y)-K_{1}(Z \mid Y)
$$

or simply

$$
\begin{aligned}
\int K(Z \mid X) T(X \mid Y) d X & =\sum_{2}^{\infty} K_{m}(Z \mid Y) \\
& =K(Z \mid Y)-K_{1}(Z \mid Y) \\
K(Z \mid Y) & =T(Z \mid Y)+\int K(Z \mid X) T(X \mid Y) d X
\end{aligned}
$$

That is, the density of arrivals near $Z$ is the sum of the density in exactly one step (given by $T(Z \mid Y)=K_{1}(Z \mid Y)$ ) plus the contribution of walks that go from $Y$ to $X$ in one step and then from $X$ to $Z$ in any additional number of steps, integrating over the intermediate position, $X$.

The recursive equation for the diffusive Green's function, Eq (2.6) has the structure of the last equation (noting, however, that its kernel has two parts corresponding to leakage across the surface and to absorption.) Thus we may construct a random walk that samples $G\left(\vec{R}, \vec{R}_{0} ; t\right)$ by a random walk in which $-\vec{n} \cdot \vec{\nabla} G_{k}\left(\vec{R}^{\prime}, \vec{R}_{k} ; t^{\prime}\right)$ or $U_{k} G_{k}\left(\vec{R}^{\prime}, \vec{R}_{0} ; t^{\prime}\right)$ are sampled for successive positions. 


\section{SAMPLING THE GREEN'S FUNCTION}

The essence of the procedure is that at every stage, the walker is at some position $\vec{R}_{k}$. We select some subdomain $\Omega_{k}$ with $\vec{R}_{k} \in \Omega_{k} \subset \Omega$. In general $\Omega_{k}$ will be a member of some class of domains (spheres, rectangular parallelepipeds, cylindrical annuli, etc.) and we will select the largest that fits inside $\Omega$. Given $\Omega_{k}$, we construct $G_{k}\left(\vec{R}, \vec{R}_{k} ; t\right)$ and sample it for a point of artificial absorption inside $\Omega_{k}$ or for a point on $\partial \Omega_{0}$ at which a walker escapes. At each step of the walk, a partial contribution to the full $G\left(\vec{R}, \vec{R}_{0} ; t\right)$ is available, namely $G_{k}\left(\vec{R}, \vec{R}_{0} ; t\right)$. We may use that to evaluate partial contributions to integrals over the full solution, or to generate random positions drawn from $G\left(\vec{R}, \vec{R}_{0} ; t\right)$.

The details of the random walk are outlined below:

(1) Initialize:

$$
k \leftarrow 0 ; \quad t_{k} \leftarrow 0 ; \quad \vec{R}_{k} \leftarrow \vec{R}_{0} ; \quad \Omega_{k} \leftarrow \Omega_{0}
$$

(2) Use $G_{k}\left(\vec{R}, \vec{R}_{0} ; t\right)$ as contribution to $G\left(\vec{R}, \vec{R}_{0} ; t\right)$.

(3) Sample $\left(\vec{R}_{k+1}, t_{k+1}\right)$ from either

(3.1) $U_{k} G_{k}\left(\vec{R}_{k+1}, \vec{R}_{k} ; t_{k+1}-t_{k}\right)$ and then

(3.11) Continue walk with probability $1-A\left(\vec{R}_{k+1}\right) / U_{k}$

or else

(3.12) terminate the walk;

or else sample next $\left(\vec{R}_{k+1}, t_{k+1}\right)$ from

(3.2) $-\vec{n} \cdot \vec{\nabla} G_{k}\left(\vec{R}_{k+1}, \vec{R}_{k} ; t_{k+1}-t_{k}\right)$ on $\partial \Omega_{k}$; if $\vec{R}_{k+1} \in \partial \Omega$ terminate the walk.

(4) Update indexes and coordinates:

$$
k \leftarrow k+1 ; \quad t_{k} \leftarrow t_{k+1} ; \quad \vec{R}_{k} \leftarrow \vec{R}_{k+1} ; \quad \Omega_{k} \leftarrow \Omega_{k+1}
$$

(5) Repeat from step (2) until walk terminates.

Eq (2.2) shows that events (3.1) and (3.2) above are mutually exclusive.

\section{USING CARTESIAN PRODUCT SUBDOMAINS}

Use of the ideas presented above are substantially advanced by the use of subdomains that are Cartesian products of sets in still lower dimensions. An example is a rectangle, $\left[x_{1} \leq x \leq x_{2}\right] \otimes\left[y_{1} \leq y \leq y 2\right]$ in which the set of points in two dimensions is specified as the Cartesian or outer product $(\otimes)$ of two intervals in one dimension. We now consider the use of subdomains $\Omega_{k}$ that are such Cartesian products:

$$
\Omega_{k}=\omega_{1}\left(\vec{R}_{k}\right) \otimes \omega_{2}\left(\vec{R}_{k}\right) \otimes \cdots \omega_{M}\left(\vec{R}_{k}\right)
$$

The intervals are shown as functions of the interior point $\vec{R}_{k}$. We might use three one-dimensional intervals to specify a rectangular parallelepiped, or the product of a twodimensional ring by a one-dimensional interval to get a truncated annulus, or the product of $N$ three-dimensional spheres to describe a finite volume in a $3 N$-dimensional space. 
The important point is that the diffusion equation in the form $\mathrm{Eq}$ (2.5) is separable. That is, if for a lower-dimensional domain $\omega_{l}$ we can find the necessary Green's function (without absorption):

$$
\begin{aligned}
{\left[-\nabla^{2}+\frac{\partial}{\partial t}\right] g_{l}\left(x_{l}, x_{0 l} ; t\right) } & =0 ; \quad x_{l}, x_{0 l} \in \omega_{l} \\
g_{l}\left(x_{l}, x_{0 l} ; 0\right) & =\delta\left(x_{l}-x_{0 l}\right) \\
g_{l}\left(x_{l}, x_{0 l} ; t\right) & =0 ; \quad x_{l}, x_{0 l} \notin \omega_{l}
\end{aligned}
$$

then

$$
G_{k}\left(\vec{R}, \vec{R}_{k} ; t\right)=\exp \left(-U_{k} t\right) \prod_{l}^{M} g_{l}\left(x_{l}, x_{0 l} ; t\right)
$$

The proof follows by straightforward computation using $\mathrm{Eq}(5.2)$.

We also have

$$
-\vec{n} \cdot \vec{\nabla} G_{0}\left(\vec{R}, \vec{R}_{0} ; t\right)=\exp \left(-U_{k} t\right) \sum_{l}\left[-\nabla_{l n} g_{l}\right] \prod_{j \neq l} g_{l}\left(x_{l}, x_{0 l} ; t\right)
$$

where $\nabla_{l n} g_{l}$ denotes the outer normal derivative of $g_{l}\left(x_{l}, x_{0 l} ; t\right)$ at the boundary of $\omega_{l}$.

The use of such Green's functions may best be described in a procedural way:

(1) Sample $\tau_{0}$ from the probability distribution function (pfd) $U_{k} e^{-U_{k} \tau_{0}}$

(2) For $l=1, \cdots, M$ sample $\tau_{l}$ from the pdf $\int_{\partial \omega_{l}}\left[-\nabla_{l n} g_{l}\left(x_{l}, x_{0 l} ; \tau\right) d x_{l}\right.$

(3) Set $l=\min \left(\tau_{0}, \tau_{1}, \cdots, \tau_{M}\right)$

if $t=\tau_{0}$ then new $R_{k} \in \Omega_{k}$

that is, each $x_{j}$ is sampled on the interior of its $\omega_{j}$ using $g_{j}\left(x_{j}, x_{0 j} ; t\right)$.

else: new $R_{k} \in \partial \Omega_{k}$

that is, $x_{l}$ is put on $\partial \omega_{l}$, the others on interior points

of their respective domains $\omega_{j}$.

\section{GREEN'S FUNCTIUNS FROM EIGENFUNCTIONS}

A practical way of writing down any of the Green's functions cited above is to use eigenfunction expansions. A general approach starts with Green's function defined in Eq(1.2). Consider $\phi_{m}(\vec{R})$, normalized eigenfunctions of the operator $-\nabla^{2}+A(\vec{R})$ :

$$
\left[-\nabla^{2}+A(\vec{R})\right] \phi_{m}(\vec{R})=\lambda_{m} \phi_{m}(\vec{R})
$$

Then we may express Green's function for the operator $-\nabla^{2}+A(\vec{R})+\frac{\partial}{\partial t}$ as

$$
G\left(\vec{R}, \vec{R}_{0} ; t\right)=\sum_{m} e^{-\lambda_{m} t} \phi_{m}(\vec{R}) \phi_{m}\left(\vec{R}_{0}\right)
$$

Straightforward application of $\mathrm{Eq}(6.1)$ and differentiation with respect to $t$ shows that it satisfies the first line of $\mathrm{Eq} 1.2$. At $t=0$ it reduces to 


$$
G\left(\vec{R}, \vec{R}_{0} ; 0\right)=\sum_{m} \phi_{m}(\vec{R}) \phi_{m}\left(\vec{R}_{0}\right)=\delta\left(\vec{R}-\vec{R}_{0}\right)
$$

Expansions for the Green's functions defined in $\mathrm{Eq}$ ( 5.2) can often be written in terms of elementary functions. For example, for a (three-dimensional) sphere of radius $a$,

$$
\phi_{m}(r) \propto \sin (m \pi r / a) / r
$$

with eigenvalues

$$
\lambda_{m}=\frac{m^{2} \pi^{2}}{a^{2}}
$$

and Green's function is

$$
g(r, t)=\frac{1}{2 a^{3}(r / a)} \sum_{m=1}^{\infty} \sin (m \pi r / a) \exp \left(-m^{2} \pi^{2} t / a^{2}\right)
$$

Note that the dimensionless function $a^{3} g$ depends only on the dimensionless variables $u=r / a$ and $\tau=t / a^{2}$. This reduces spheres of all possible radii to one case.

The expansion of $\mathrm{Eq}$ (6.6) converges slowly at small $t$. There is a complementary expansion that is in effect an "image" expansion and that converges rapidly at short time. Formally it derives from the Poisson sum rule ([2], p. 275) and it is

$$
g(r, t)=\left\{c^{-u^{2} /(4 \tau)}+\sum_{m=1}^{\infty}\left[(u+2 m) e^{\left.-(u+2 m)^{2} /(4 \tau)+(u-2 m) e^{-(u-2 m)^{2} /(4 \tau)}\right]}\right\} /(4 \pi \tau)^{3 / 2}\right.
$$

Green's function for a one-dimensional interval $\left[x_{1}, x_{2}\right]$ is similar. Because it is translationally invariant, we nced only write it down on some canonical interval, say $-a \leq x, x_{0} \leq a$ where it takes the form

$$
g\left(x, x_{0} ; t\right)=\frac{1}{a} \sum_{m=1}^{\infty} \sin \left[\frac{m \pi(x+a)}{2 a}\right] \sin \left[\frac{m \pi\left(x_{0}+a\right)}{2 a}\right] \exp \left[-\left(\frac{m \pi}{2 a}\right)^{2} t\right]
$$

Using reduced variables, $u=(x-x 0) / a ; \tau=t / a^{2}$ the short-time expansion, corresponding to $\mathrm{Eq}(6.7)$ is

$$
a g\left(x, x_{0} ; t\right)=\sum_{m=-\infty}^{\infty} \frac{e^{-(u+2 m)^{2} /(4 \tau)}}{(4 \pi \tau)^{1 / 2}}
$$

For cylindrical coordinates, we may consider the interval $a \leq r \leq b$ for which the basis functions are (cf ref [2], p. 206.)

$$
\Phi_{m}(r)=\pi \alpha_{m} J_{0}\left(\alpha_{m} a\right) \frac{J_{0}\left(\alpha_{m} r\right) Y_{0}\left(\alpha_{m} b\right)-J_{0}\left(\alpha_{m} b\right) Y_{0}\left(\alpha_{m} r\right)}{2\left(J_{0}^{2}\left(\alpha_{m} a\right)-J_{0}^{2}\left(\alpha_{m} b\right)\right)^{1 / 2}}
$$

where $J_{0}(x)$ is the Bessel function of the first kind of order zero, and $Y_{0}(x)$ the Bessel function of the second kind of order zero. The eigenvalues are $\lambda_{m}=\alpha_{m}^{2}$ where $\alpha_{m} q$ are solutions of

$$
J_{0}\left(\alpha_{m} a\right) Y_{0}\left(\alpha_{m} b\right)-J_{0}\left(\alpha_{m} b\right) Y_{0}\left(\alpha_{m} a\right)=0
$$


so that the Green's function we need is

$$
g_{c y l}\left(r, r_{0} ; t\right)=\sum_{m} e^{-\alpha_{m}^{2} t} \Phi_{m}(r) \Phi_{m}\left(r_{0}\right)
$$

If $b / a-1$ is not large then

$$
\Phi_{m}(r) \approx\left[\frac{2}{b-a}\right)^{1 / 2} \sin [m \pi(r-a) /(b-a)]
$$

and

$$
\alpha_{m}(r) \approx \frac{a \pi}{b-a}
$$

This can be used very well as the starting point for a rapidly converging iterative solution of $\mathrm{Eq}$ (6.11). Also, Eq (6.14) means that for most zones, computation with sines and cosines can replace use of the Bessel functions.

\section{SAMPLING THE FUNCTIONS}

We need to consider how to sample new positions and time intervals from $g_{l}\left(x_{l}, x_{0 l} ; t\right)$ and $\int_{\partial \omega_{l}}\left[-\nabla_{l n} g_{l}\left(x, x_{0 l} ; t\right)\right] d x$, respectively. As a basis for experimentation, we prepared robust but not optimally efficient routines for the purpose. For the positions in the interior of the subdomains, we used rejection lechniques based the leading terms of the short and long time expansions. For sampling times to boundary absorption, we proceed as follows: Denote

$$
H_{l}(t)=\int_{\omega_{l}} g_{l}\left(x, x_{0 l} ; t\right) d x
$$

It follows from $\mathrm{Eq}(5.2)$ that

$$
\begin{aligned}
H_{l}^{\prime}(t) & =\int_{u_{l} l} \frac{\partial g_{l}\left(x, x_{0 l} ; t\right)}{d t} d x \\
& =-\int_{\partial \omega_{l}} \nabla_{l n} g_{l}\left(x, x_{0 l} ; t\right) d x
\end{aligned}
$$

It is straightforward to compute $H_{l}(t)$ (in appropriate dimensionless form) from sort or long time series and to tabulate the results in a form straightforward to sample. These routines exist in usable form. They have been tested using soluble test problems. They have not been tested in the context of radiation transport. The plan is to test the efficiency of the calculation when the sphere construction presently used is replaced by rectangular and/or cylindrical domains. The expectation is that the better fit of the new shapes now available will speed up the calculations. This will be very much application dependent. On the other hand, the use of boxes or cylinders is somewhat more expensive, requiring sampling for each of the orthogonal directions, and more complicated geometry to orient the subdomain. It may be that a speedup will be attained only when the existing routines are made more efficient, but there is significant room to do that. 


\section{REFERENCES}

[1] M.H. Kalos, D. Levesque, and L. Verlet, "Helium at 'Lero 'lemperature with Hard Sphere and Other Forces", Physical Review A 9 (1974).

[2] H.S. Carslaw and J.C. Jaeger, "Conduction of Heat in Solids", Clarendon Press, (Oxford, 1959), p. 275. 
Although this term could be made small by choosing $\Delta \tau$ small enough, we do not do so in order to find the "worst case scenario." Hence, ignoring $1 / \Lambda \tau$,

$$
\begin{aligned}
\lim _{(c \Delta t)-r 0} \Delta S_{i} & <+p / c_{\mathrm{v}, i} \\
& =\left(S_{i}^{\prime} / \bar{S}_{i}^{\prime}\right)\left(\bar{c}_{\mathrm{v}, i} / c_{\mathrm{v}, i}\right)\left(S_{0}-S_{i}\right) \\
& <\left(S_{i}^{\prime} / \bar{S}_{i}^{\prime}\right)\left(\bar{c}_{\mathrm{v}, i} / c_{\mathrm{v}, i}\right) S_{0} .
\end{aligned}
$$

Since $\bar{S}_{i}^{\prime}$ is obtained at an intermediatc point between $S_{0}$ and $S_{i},\left(S_{i}^{\prime} / \bar{S}_{i}^{\prime}\right)<1$. For an ideal gas, $\bar{c}_{\mathrm{v}, i} / c_{\mathrm{v}, i}=1$; hence, $\Delta S_{i}<S_{0}$. As long as $c_{\mathrm{v}, i}$ grows no faster than $T^{3}$, we still get $\left(S_{i}^{\prime} / \bar{S}_{i}^{\prime}\right)\left(\bar{c}_{\mathrm{v}, i} / c_{\mathrm{v}, i}\right) \leq 1$ and the lemma is proved. ${ }^{1}$ To summarize, we stress that the condition $\Delta S_{i}<S_{0}$ is a worst case. Under normal conditions, we expect $\Delta S_{i}<0$ as desired. I

Because the iterates satisfy a discretization of (24), the densities $E_{i}$ and $\rho e_{i}$ satisfy a conservation law. It is easy to see what contributes to the radiation energy change, $V\left(E_{i+1}-E_{i}\right)$, for each iterate in each cell. In particular the term,

$$
\Delta \tau[\mathcal{K}(S-E)]_{i+1} \doteq \Delta \tau\left[\mathcal{K}_{i}\left(S_{i+1}-E_{i+1}\right)+\mathcal{K}_{i}^{\prime}\left(S_{i}-F_{i i}\right)\left(S_{i+1}-S_{i}\right)\right]
$$

is the energy transferred from the matter. In that light, energy is conserved by setting,

$$
e_{i+1}=e_{i}+\Delta \tau\left\{e_{0}-e_{i}-\frac{c_{\mathrm{v}, i}}{S_{i}^{\prime}}\left(S_{i+1}-S_{i}\right)-[\mathcal{K}(S-E)]_{i+1}\right\}
$$

where the coupling term is defined in (41) If (42) should ever yield $e_{i+1}<0$, we easily recover by using the EOS: $e_{i+1}=e\left(T_{i+1}\right)$ where $T_{i+1}=\left(S_{i+1} / a\right)^{1 / 4}$.

We conclude the $\Psi$ tc analysis with two more items.

\section{1 $\Psi$ tc for large $\Delta t$}

Setting the real time step $\Delta t$ very large effectively discards the first term on the rhs of (10) and (11). In this case, the $\Psi$ tc iterations are very robust. Equation (28) becomes,

$$
-\mathcal{D}_{i} E_{i+1}+\left(V / \Delta \tau+\alpha_{i} \mathcal{K}_{i}\right) E_{i+1}=(V / \Delta \tau) E_{i}+\alpha_{i} \mathcal{K}_{i} S_{i}
$$

while (29) becomes,

$$
S_{i+1}=\left(1-\gamma_{i}\right) S_{i}+\gamma_{i} E_{i+1},
$$

where $\alpha_{i}$ and $\gamma_{i}$ are defined as before, but now $a_{i}=m c_{\mathrm{v}, i} V / \Delta \tau$. Clearly, there is no danger of unphysical iterates.

However, in most applications, even though $\Delta t$ may be large, the two terms on the rhs of (11) may be comparable, especially if the temperatures are cold. For example, for hydrogen, if $T$ is measured in $\mathrm{keV}, e=0.1 T$ while $E \approx S=0.0137 T^{4}$. Hence, we should also analyze the effects of only discarding $\partial_{t} E$. In this regime, on the lhs of $(28), g_{i} \rightarrow V / \Delta \tau+\alpha_{i} \mathcal{K}_{i}$ while on the rhs the term $V E_{0}$ is discarded. Equation (29) and the definitions (27) are unchanged. The previous analysis holds, but $\Delta \tau$ might be restricted further because on the rhs of (28), which must still be kept positive, we lose the term $V E_{0}$. Also, the system is less diagonally dominant because $g_{i}$ is smaller. Nevertheless, $\Delta \tau$ still exerts a stabilizing effect on the iterations.

\subsection{Conclusion of $\Psi$ tc iterations}

In a sense, determining when to stop iterating is trivial. The answer is obtained when $S_{i+1}=S_{i}$ and $E_{i+1}=E_{i}$. However, in order to have an efficient termination criterion, we focus attention on when the matter energy stops changing. First, we compute the maximum energy in a cell, at the $i^{\text {th }}$ step

$$
(m e)_{i, x}=\max _{\text {cells }}\left(m e_{i}\right)
$$

\footnotetext{
${ }^{1}$ For the Pomraning problem, since $c_{\mathrm{v}, i} \propto T^{3},\left(S_{i}^{\prime} / \bar{S}_{i}^{\prime}\right)\left(\bar{c}_{\mathrm{v}, i} / c_{\mathrm{v}, i}\right)=1$.
} 
and stop whenever

$$
\frac{m\left(e_{i}-e_{i-1}\right)}{m e_{i}+\epsilon_{1}(m e)_{i, x}+\epsilon_{2}(m e)_{0}}<\epsilon_{3}
$$

where $(m e)_{0}$ is a user-specified reference energy and the $\epsilon_{i}$ are user specified parameters. The criterion halts the iterations if either the relative energy change, or two comparisons of the absolute change are small. Suggested values are $\epsilon_{1}=\epsilon_{2}=0.001$ and $\epsilon_{3}=10^{-8}$.

Of course, halting when the matter energy has converged is not the whole story, but typically the matter energy is larger than the radiation energy. Nevertheless, if (43) is satisfied at the $i^{\text {th }}$ step, the next iteration for $E_{i+1}$ is (28) where we explicitly write $\mathcal{D}_{i}=\mathcal{D}\left(E_{i}, S_{i}\right)$ to show that the diffusion coefficient depends on both $E_{i}$ and $S_{i}$. If we now assume that $S_{i+1}=S_{i}$, then $e_{i+1}=e_{i}$ and the previous iteration is of the form,

$$
-\mathcal{D}\left(E_{i-1}, S_{i}\right) E_{i}+g_{i} E_{i}=(V / \Delta \tau) E_{i-1}+V E_{0}+\alpha_{i} \mathcal{K}_{i} S_{i}+\left(1-\alpha_{i}\right) m\left(e_{0}-e_{i}\right)
$$

Subtracting (44) from (28) yields,

$$
-\left[\mathcal{D}\left(E_{i}, S_{i}\right) E_{i+1}-\mathcal{D}\left(E_{i-1}, S_{i}\right) E_{i}\right]+g_{i} \Delta E_{i}=(V / \Delta \tau) \Delta E_{i-1}
$$

where $\Delta E_{i}=E_{i+1}-E_{i}$. The different diffusion coefficients stem from the flux limiter. This difference may be ignored since the flux limiter is only a kludge. After dividing by $\gamma_{i}$, (45) becomes

$$
-\left(1 / g_{i}\right) \mathcal{D}_{i} \Delta E_{i}+\Delta E_{i}=h_{i} \Delta E_{i-1}
$$

where $0<h_{i}=(V / \Delta \tau) / g_{i}<1$. This is an elliptic equation with homogeneous boundary conditions. The rhs is a fraction of what is presumably already a small quantity $\Delta E_{1-1}$. Hence, $\Delta E_{i}$ is smaller yet and the $E$ equation may also be deemed to have converged.

If by chance we halted promaturely, there is one more control available. If (24) is viewed as a system of evolution equations, the coefficients have "characteristic times" that govern how long (in pseudo time) it takes to equilibrate. If (24) is divided by the coll volume $V$, the units are cncrgy-density/pseudo-time. If $\tau$ is the approximate time to equilibrate, then from the equation and the absorption term, e/ $\tau \approx-e$ which yields $\tau \approx 1$. Similarly, from the coupling term,

$$
m e / \tau \approx \mathcal{K} S=c \Delta t m \kappa S
$$

which yields,

$$
\tau \approx e /(c \Delta t \kappa S)
$$

as another estimate for how long (in pscudo time) to iterate.

\section{Pomraning Problem}

We now present results on the Pomraning problem, [2] and [3], which simulates a radiation wave propagating into cold material. After some algebra, for this problem, the equations may be put in the form,

$$
\begin{aligned}
\partial E / \partial \tilde{t} & =\nabla \cdot D \nabla E+(1 / \epsilon)(S-E) \\
\partial e / \partial \tilde{t} & =-(1 / \epsilon)(S-E) .
\end{aligned}
$$

where $D=1 /(3 \epsilon), e=S / \epsilon$, and $S=a T^{4}$. The term $\epsilon$ is a constant which arises by using $c_{\mathrm{v}}=c_{\mathrm{v}, 0} T^{3}$ and setting $\epsilon=4 a / c_{\mathrm{v}, 0}$. In (46)-(47), $\kappa=\rho=1$. Hence, $e=\rho e$ is now the internal energy density. 'I'he normalized time $\tilde{t}=\epsilon c \kappa t$.

'The specifications of the Pomraning problem dictate $S \leq E$. Since (46)-(47) imply that $\epsilon$ is an approximate time for the fields to equilibrate, it is interesting to examine what happens at early times using the backward Euler $(\theta=1)$, semi-implicit scheme described in $\S 2$. 'lable 1 displays the energy 


\begin{tabular}{|cc|ccccc|}
\hline$\Delta \tilde{t}_{0}$ & $t$ & $j=0$ & 1 & 2 & 3 & 4 \\
\hline $1 . \mathrm{e}-7$ & $1 . \mathrm{e}-7$ & $-1.5 \mathrm{e}+7$ & $-1.6 \mathrm{e}-3$ & $9.4 \mathrm{e}-10$ & $2.5 \mathrm{e}-12$ & $6.1 \mathrm{e}-15$ \\
$1 . \mathrm{e}-8$ & $1 . \mathrm{e}-8$ & $-1.5 \mathrm{e}-1$ & $3.2 \mathrm{e}-8$ & $9.6 \mathrm{e}-13$ & $2.6 \mathrm{e}-16$ & $6.2 \mathrm{e}-20$ \\
$1 . \mathrm{e}-9$ & $1.14 \mathrm{e}-8$ & $1.1 \mathrm{e}-5$ & $2.4 \mathrm{e}-9$ & $3.4 \mathrm{e}-13$ & $3.6 \mathrm{e}-17$ & $3.1 \mathrm{e}-21$ \\
$1 . \mathrm{e}-10$ & $1.09 \mathrm{e}-8$ & $1.1 \mathrm{e}-5$ & $2.0 \mathrm{e}-9$ & $2.4 \mathrm{e}-13$ & $2.1 \mathrm{e}-17$ & $1.4 \mathrm{e}-21$ \\
\hline
\end{tabular}

Table 1: Energy exchange term $E_{i}-S_{i}$ for the Pomraning problem. First and second columns denote the initial time step and current time resp. Simulation uses $\epsilon=0.1$

exchange term $E_{j}-S_{j}$ where $j$ denotes the mesh index. A nonuniform mesh is used; $\Delta z_{0}=0.01$, and for $j>0, \Delta z_{j}=\Delta z_{0}(1.05)^{j}$. The table shows that for $\Delta \tilde{t}_{0}=10^{-8}$, at the incident edge, $S_{0}>E_{0}$ violating the physics. For larger $\Delta \tilde{t}_{0}$, the result is even worse, e.g., if $\Delta \tilde{t}_{0}=10^{-6}$, after one cycle, $E_{0}-S_{0}--1.2 \cdot 10^{15}$

We note that the above error does not occur for the proposed $\Psi$ tc scheme. However, the lack of the error is not due to the introduction of the pseudo-temporal derivative, but is instead due to iterating on $S$ instead of $T$. Indeed, since the opacity $\kappa$ is constant, for the first iteration, the pseudo-time step $\Delta \tau$ is not restricted; hence, $1 / \Delta \tau=0$. Equation (34) still holds and for the first time step of the Pomraning problem, it reduces to,

$$
S_{1}=\gamma_{0} E_{1} \quad \gamma_{0}=1 /\left(1+\alpha_{0} \mathcal{K}_{0} / V\right),
$$

where the subscripts now denote the iteration number and where $\alpha_{0}=1 /(1+c \Delta t \kappa \epsilon)=1 /(1+\Delta \tilde{t})$. This is the desired behavior; viz., $S_{1} \leq E_{1}$ with equality obtained only as $\Delta \tilde{t} \rightarrow \infty$.

Iterating on $S$ (instead of $T$ ) is the cause of the good fortune. Substituting $\Delta T=\Delta S / S_{0}^{\prime}$ into (8) yields

$$
\lim _{\Delta t \rightarrow \infty} \Delta T=\lim _{\Delta t \rightarrow \infty} \Delta S / S_{0}^{\prime}=\left(E-S_{0}\right) / S_{0}^{\prime}
$$

and we obtain $S=E$ as desired.

The analysis is easily extended to other problems with more general material properties, e.g., $c_{v}=$ $c_{\mathrm{v}, 0} T^{\beta}$ and $\kappa=\kappa_{0} T^{\delta}$. The moral is that since $S \propto T^{4}$, it is better to solve for (and iterate on) what varies rapidly $(S)$, then take its fourth root (to get $T$ ) instcad of using $T$ and then taking its fourth power.

\section{Conclusion}

We have developed a scheme to solve the nonlinear system of equations describing radiation diffusion coupled to matter energy balance. Because of the nonlinearities, iterations are required. After multiplying by the time step $\Delta t$, the equations mimic a coupled system with transport, explicit sources and absorption. By introducing $\Psi$ tc, the system is solved when the (pseudo-time) steady state is reached. Each iteration is a temporal advance in pseudo-time and requires only one solve of a scalar elliptic equation. The scheme is robust; by controlling the pseudo-time step $\Delta \tau$, each iterate is guaranteed to be positive and bounded by the previous iterate and the boundary conditions. The solution is first order accurate in (real) time. Arbitrarily large $\Delta t$ 's are allowed. Upon convergence, all of the coefficients are evaluated at the advanced time level.

Acknowledgement Several results in this paper stem from useful discussions with Dr. J. Bolstad of LLNL. 


\section{References}

[1] A. I. Shestakov, J. L. Milovich, and M. K. Prasad, "Combining Cell and Point Centered Methods in 3D, Unstructured-Grid Radiation-Hydrodynamic Codes," submitted to J. Comput. Phys., available from shestakov@llnl.gov.

[2] G. C. Pomraning, JQSRT, 21, 249 (1979)

[3] B. Su and G. L. Olson, JQSRT, 56, 3, 337 (1996)

[4] C. T. Kelley and D. E. Keyes, "Convergence Analysis of Pseudo Transient-Continuation," SLAM J. Num. Anal., 35, 508-523 (1998).

[5] G. J. Pert, "Physical Constraints in Numerical Calculations of Diffusion," J. Comp. Phys., 42, (1981) p. 20 